\title{
Interindividual Differences and Intraindividual Variability in the Cortisol Awakening Response: An Examination of Age and Gender
}

\author{
David M. Almeida, Jennifer R. Piazza, and Robert S. Stawski \\ Pennsylvania State University
}

\begin{abstract}
This article shows age and gender differences in the magnitude and day-to-day variability of the cortisol awakening response (CAR) using a national sample of 1,143 adults who completed the second wave of the National Study of Daily Experiences, a part of the Midlife Development in the United States survey. Participants between the ages of 33 and 84 years completed 8 consecutive nightly interviews and provided 4 saliva samples (upon waking, $30 \mathrm{~min}$ after waking, before lunch, and before bed) on 4 consecutive interview days. Results revealed substantial day-to-day variability in the CAR as well as significant Age $\times$ Gender interactions, indicating that although no systematic age-related differences emerged for women, the magnitude and day-to-day variability of the CAR increased with age among men.
\end{abstract}

Keywords: multilevel modeling, HPA axis, stress

The cortisol awakening response (CAR) has garnered a great deal of attention in recent years (see review by Clow, Thorn, Evans, \& Huckelbridge, 2004). Referring to the change in cortisol that occurs within the first hour after awakening, the CAR exhibits a $20 \%-50 \%$ increase at approximately $30-45$ min postawakening and begins a gradual descent thereafter (Chahal \& Drake, 2007). Although the function of the CAR is not entirely understood, some researchers posit that it may prepare an organism for engagement with the external environment (Fries, Dettenborn, \& Kirschbaum, 2009; Pruessner et al., 1997). Other researchers, however, suggest that its primary importance may lie in regulating the immune system (e.g., Hucklebridge, Clow, Abeyguneratne, Huezo-Diaz, \& Evans, 1999). Despite the uncertainty of the CAR's precise physiological role, it is clear that alterations in this pattern are associated with several adverse outcomes. For example, blunted CAR has been associated with burnout symptomatology (Pruessner, Hellhammer, \& Kirschbaum, 1999) and posttraumatic stress disorder (Wessa, Rohleder, Kirschbaum, \& Flor, 2006), whereas heightened CAR has been associated with depressive symptomatology (e.g., Bhagwagar, Hafizi, \& Cowen, 2003) and neuroticism (Portella, Harmer, Flint, Cowen, \& Goodwin, 2005).

Early research on the CAR revealed a high degree of intraindividual stability in this response, and researchers primarily focused their attention on investigating interindividual differences in the magnitude of this response (e.g., Pruessner et al., 1997; see reviews by Schulz, Kirschbaum, Pruessner, \& Hellhammer, 1998;

David M. Almeida, Jennifer R. Piazza, and Robert S. Stawski, Department of Human Development and Family Studies, Pennsylvania State University.

Research reported in this article was supported by National Institute on Aging Grants P01 AG020166 and R01 AG019239 to conduct a longitudinal follow-up of the MIDUS (Midlife in the United States) investigation.

Correspondence concerning this article should be addressed to David M. Almeida, Department of Human Development and Family Studies, Pennsylvania State University, 118 Henderson Building, University Park, PA 16802. E-mail: dalmeida@psu.edu
Wüst et al., 2000). In recent years, however, researchers have begun to examine intraindividual variability (i.e., day-to-day fluctuations) in the CAR (e.g., Dahlgren, Kecklund, Theorell, \& Åkerstedt, 2009; de Weerth \& Buitelaar, 2005; Kunz-Ebrecht, Kirschbaum, Marmot, \& Steptoe, 2004; Stalder, Hucklebridge, Evans, \& Clow, 2009; Thorn, Hucklebridge, Evans, \& Clow, 2009). The current study extends previous research by examining age and gender differences in the magnitude and day-to-day variability of the CAR across 4 consecutive days. We examined these differences in a national sample of 1,143 adults between the ages of 33 and 84 years.

\section{The CAR}

Cortisol, a hormone secreted by the hypothalamic-pituitaryadrenal (HPA) axis, exhibits a fairly robust diurnal pattern, peaking within an hour after waking and declining thereafter, until reaching a nadir at approximately midnight (Fries et al., 2009). The initial rise in cortisol, referred to as the cortisol awakening response, is a distinct component of the diurnal pattern of cortisol (see review by Clow et al., 2004) and is considered to be a marker of the integrity of the HPA axis (Hellhammer et al., 2007). Values of the CAR vary across studies, but an initial increase within 30-min postawakening appears to be relatively consistent (see review by Clow et al., 2004). Exhibiting an absence or an exacerbation of this increase is associated with several adverse psychological and physiological outcomes, including burnout (Pruessner et al., 1999), neuroticism (Portella et al., 2005) and health problems (Kudielka \& Kirschbaum, 2003) such as persistent pain (Geiss, Varadi, Steinbach, Bauer, \& Anton, 1997) and hypertension (Wirtz et al., 2007).

\section{Intraindividual Variability in the CAR}

Until recently, researchers have focused primarily on investigating the magnitude of the CAR on one day or by averaging the CAR across multiple days. Although this research is highly informative, we believe there is also much to be gained by examining intraindividual variability in the CAR, typically characterized by 
the amount of day-to-day fluctuation in this response. Some researchers have cautioned that despite safeguards used to check compliance, intraindividual variability in the CAR may still primarily reflect measurement error (Kudielka, Broderick, \& Kirschbaum, 2002). Although this may partially be the case, intraindividual variability in the CAR may also reflect other systematic changes in the HPA axis. Indeed, just as variability in psychological measures has been linked to long-term negative outcomes (Eizenmann, Nesselroade, Featherman, \& Rowe, 1997), intraindividual variability in the CAR may also be associated with substantive outcomes. Researchers beginning to explore this idea have shown that the CAR is, in fact, influenced by both state and trait measures (Hellhammer et al., 2007), including factors such as seasonality (Thorn et al., 2009) and time of awakening (e.g., Federenko et al., 2004; Stalder et al., 2009; Williams, Magid, \& Steptoe, 2005). Continuing this line of inquiry, we examined the extent to which important demographic variables are simultaneously associated with magnitude and day-to-day variability in the CAR.

One particular challenge in assessing intraindividual variability in the CAR is appropriately modeling the multiple sources of variation in this response. Multilevel models, also referred to as hierarchical linear models (Raudenbush \& Bryk, 2002; Snijders \& Bosker, 1999), are designed to accommodate nested data, such as those found in daily diary studies (Nezlek, 2008). These models can be used to assess variability in the magnitude of the CAR between individuals (i.e., interindividual variability) as well as day-to-day variability across time (i.e., intraindividual variability). Oftentimes, such models are employed to specifically consider individual differences or heterogeneity in mean levels of some variable, such as whether the magnitude of the CAR differs by age or gender. However, these models can be extended to consider individual differences or heterogeneity in intraindividual variability. Hoffman (2007) provides a very useful tutorial that demonstrates how individual differences in both inter- and intraindividual variability can be modeled simultaneously. Such an approach avoids confounds between magnitude and variability, as they are considered and modeled simultaneously. Furthermore, drawing on the modeling framework described by Hoffman (2007), we can examine age and gender differences in the magnitude and day-today variability of this response.

\section{Age, Gender, and the CAR}

There is a $20 \%-50 \%$ increase in basal cortisol levels between the ages of 20 and 80 (see review by Chahal \& Drake, 2007). It is unclear, however, whether there are age differences in the CAR, as research examining this response has revealed mixed findings. Although some studies have revealed age-related increases in the CAR (e.g., Kudielka \& Kirschbaum, 2003), others have found no such age differences (e.g., Wüst et al., 2000). Making generalizations about this response, then, is difficult, as blunted or heightened responses may differ as a function of age. For example, what could be considered a heightened response for younger adults may actually be a normal response for older adults. In addition, it is unclear whether intraindividual variability in the CAR increases or decreases as a function of age.

Findings regarding gender differences in the CAR are also mixed, with some studies reporting an increased response among women and other studies showing no such gender differences (see review by Clow et al., 2004). Consequently, when examining the combined influence that gender and age may have on the CAR, the picture is murky at best. These discrepant findings may partially stem from methodological issues present across previous studies, such as too few sample days and attenuated age ranges (see review by Clow et al., 2004). Moreover, several of the studies examining age and gender differences in the CAR were based on relatively small sample sizes, so investigating these questions in a larger sample may provide adequate statistical power to help clarify these associations.

\section{The Current Investigation}

The goals of the current investigation were twofold. The first was to examine stability and lability of the CAR, operationalized as change in morning cortisol levels over the first 30 min after awakening. To this end, we decomposed the total variation of the CAR into interindividual differences in mean levels across days (i.e., betweenpersons differences) and intraindividual variability (i.e., within-person fluctuations) from day to day. The second goal was to examine the extent to which age and gender predict both magnitude and day-today variability in the CAR. We examined these questions in a national sample of adults between the ages of 33 and 84, who provided four samples of salivary cortisol across 4 consecutive days.

\section{Method}

\section{Participants}

Participants in the current study included 1,143 adults who completed the National Study of Daily Experiences, which is the daily diary portion of the second wave of the Midlife Development in the United States survey. The mean age of the sample was 57 years $(S D=12$; range: $33-84)$, and gender was approximately evenly split, with women composing $55 \%$ of the sample. Participants were fairly well educated, with $30 \%$ having a high school diploma or less, $51 \%$ having at least some college or a bachelor's degree, and $19 \%$ having at least some education beyond a bachelor's degree.

\section{Procedure and Assessment of Salivary Cortisol}

The National Study of Daily Experiences protocol consisted of eight consecutive nightly telephone interviews, in which participants were asked about their daily experiences during the previous $24 \mathrm{hr}$ (Almeida, Wethington, \& Kessler, 2002). Participants also provided saliva samples on 4 consecutive interview days. Data collection consisted of dividing respondents into separate groups, or "flights," of interviews. Each flight represented the 8-day sequence of interviews from approximately 30 respondents. Initiation of interview flights was staggered across the day of the week to control for potential confounding between day of study and day of week. Participants were compensated $\$ 25$ for their participation.

Respondents received a home saliva collection kit 1 week prior to their initial phone call. Saliva was obtained with salivette collection devices (Sarstedt, Nümbrecht, Germany). Sixteen numbered and color-coded salivettes were included in the collection kit, as was a detailed instruction sheet. Telephone interviewers reviewed the collection procedures and answered participants' questions. On Days 2-5, respondents provided four saliva samples 
per day that were later assayed for cortisol. Saliva was collected immediately upon waking, $30 \mathrm{~min}$ after waking, before lunch, and at bedtime. Cortisol concentrations were quantified with a commercially available luminescence immunoassay (IBL, Hamburg, Germany), with intra-assay and interassay coefficient of variations below five (Dressendörfer, Kirschbaum, Rohde, Stahl, \& Strasburger, 1992; Polk, Cohen, Doyle, Skoner, \& Kirschbaum, 2005). Data on the exact time respondents provided each saliva sample were obtained from the nightly telephone interviews as well as from a paper-pencil log sent with the collection kit. In addition, approximately $25 \%$ of the respondents received a "smart box" to store their salivettes (Cayuga Design, Ithaca, NY). These boxes contained a computer chip that recorded the time respondents opened and closed the box, thus providing an additional compliance check. The correlations of self-reported times across collection occasions were all above .9 . The correlations between selfreported times and times obtained from the smart box range from .75 for the evening occasion to .95 for the first morning occasion. Furthermore, the absolute mean difference between self-reported time and smart box time was $10 \min (S D=12$; range: $0-50)$ for the first morning occasion and $7 \mathrm{~min}(S D=14$; range: $0-60)$ for the second morning occasion.

\section{Control Variables}

Smoking status. Smoking status was determined by respondents identifying themselves as routine smokers as well as by the number of cigarettes individuals reported consuming on a daily basis during the study period. Individuals who did not identify themselves as smokers or who did not report smoking any cigarettes during the study protocol were classified as nonsmokers. A dichotomous variable $(0=$ nonsmoker, $1=$ smoker $)$ was used as the index of smoking status.

Medication use. Participants reported their current use of medications known to influence cortisol, including steroid inhalers, steroid medications, medications containing cortisone, birth control pills, other hormonal medications, and antidepressant or antianxiety medications (Granger, Hibel, Fortunato, \& Kapelewski, 2009). A dichotomous variable was created to indicate whether a participant reported taking any of the aforementioned medications currently $(0=$ did not use medications, $1=$ used medications $)$.

\section{Analytic Strategy}

For the purposes of the present study, we used the first two morning cortisol samples (upon waking and 30 min after waking) on each of the 4 days. Therefore, to estimate an overall growth model for the CAR, we employed a three-level multilevel model, whereby samples were nested within days, which were nested within persons (Snijders \& Bosker, 1999). As shown in Equation 1,

$$
\begin{gathered}
\text { Level 1: Cortisol } 1_{s d i}=a_{0 d i}+a_{1 d i}\left(\text { Time }_{s d i}\right)+e_{s d i} \\
\begin{aligned}
\text { Level 2: } a_{0 d i}=b_{00 i}+u_{0 d i} \\
a_{1 d i}=b_{10 i}+u_{1 d i} \\
\text { Level 3: } b_{00 i}=y_{000}+v_{00 i}
\end{aligned}
\end{gathered}
$$

$$
b_{10 i}=y_{100}+v_{10 i}
$$

at Level 1, the cortisol level for sample $s$, on day $d$, for person $i$, is a function of an intercept $\left(a_{0 d i}\right)$, which reflects the level of cortisol upon waking; a slope parameter $\left(a_{1 d i}\right)$, which reflects the change in cortisol associated per hour since waking, or the CAR slope; and a residual variance term $\left(e_{s d i}\right)$. At Level 2 , the Level 1 parameters become the outcomes whereby a person's intercept or average level of cortisol upon waking for a given $\operatorname{day}\left(a_{0 d i}\right)$ is a function of his or her average level of cortisol upon waking across study days $\left(b_{\mathrm{OO} i}\right)$, and a variance component $\left(u_{\mathrm{O} d i}\right)$, which reflects deviations from a person's average across days. Similarly, a person's CAR slope for a given day $\left(a_{1 d i}\right)$ is a function of his or her average slope $\left(b_{10 i}\right)$, and a variance component reflecting deviations from his or her average slope $\left(u_{1 d i}\right)$. At Level 3, a person's intercept, or level of cortisol upon waking $\left(b_{\mathrm{OO} i}\right)$, is a function of the sample's average level of cortisol upon waking $\left(y_{000}\right)$, and a variance component reflecting that person's deviation from the sample average $\left(v_{00 i}\right)$. Similarly, a person's CAR slope $\left(b_{10 i}\right)$ is a function of the sample's average CAR slope $\left(y_{100}\right)$, and a variance component reflecting deviations from the sample mean $\left(v_{10 i}\right)$.

To model age and gender differences in both average magnitude and intraindividual variability in the CAR slope, we used two-level multilevel models following methods outlined by Hoffman (2007). The dependent variable for the models was a CAR slope variable that was calculated by taking the difference between the two natural log-transformed cortisol samples and dividing by the duration that transpired between the samples: (InSample2 lnSample1)/(Sample2Time - Sample1Time). This variable provides an index of the change in morning cortisol levels (i.e., CAR) in natural log units per hour. This variable was put into the model shown in Equation 2:

Level 1: $\mathrm{CAR}_{d i}=a_{0 i}+a_{1 i}$ (WakeupTime $_{d i}$

$$
\text { - MeanWakeupTime } . i)+e_{d i}
$$

Level 1 residual: $\sigma_{i}^{2}=z_{0}\left\{\exp \left[z_{1}\left(\right.\right.\right.$ MeanWakeupTime $\left._{. i}\right)$

$$
\left.\left.+z_{2}\left(\operatorname{Age}_{. i}\right)+z_{3}\left(\operatorname{Gender}_{. i}\right)+z_{4}\left(\operatorname{Age} \times \operatorname{Gender}_{. i}\right)\right]\right\}
$$

Level 2: $a_{0 i}=b_{00}+b_{01}\left(\right.$ MeanWakeupTime $\left._{. i}\right)+b_{02}\left(\right.$ Age $\left._{i}\right)$

$$
\begin{gathered}
+b_{03}\left(\operatorname{Gender}_{. i}\right)+b_{04}\left(\text { Age } \times \operatorname{Gender}_{. i}\right)+u_{0 i} \\
a_{1 i}=b_{10}+u_{1 i}
\end{gathered}
$$

At Level 1, the CAR on day $d$ for person $i$ is a function of an intercept $\left(a_{0 i}\right)$, which reflects the person's CAR for that day; a person mean-centered effect of wake-up time $\left(a_{1 i}\right)$, which reflects the change in the CAR for each hour a person woke up later than usual; and a residual variance term $\left(e_{d i}\right)$. Furthermore, the Level 1 residual variance term can itself be treated as an outcome variable reflected as $\left(\sigma_{i}^{2}\right)$, as it is allowed to vary across individuals. With a log-linear model, variables can be added as predictors of this residual variance. In Equation 2, the residual variability, or intraindividual variability in the CAR, has been exponentiated to allow for linear prediction of the variance component, and is defined as a function of a person's average wake-up time across the study days $\left(z_{1}\right)$, age $\left(z_{2}\right)$, gender $\left(z_{3}\right)$, and an Age $\times$ Gender interaction $\left(z_{4}\right)$. At Level 2, a person's average CAR is given as a function of 
an intercept $\left(b_{00}\right)$, which reflects the sample average CAR, his or her average wake-up time across the study days $\left(b_{01}\right)$, his or her age $\left(b_{02}\right)$, his or her gender $\left(b_{03}\right)$, and the Age $\times$ Gender interaction $\left(b_{04}\right)$. Also at Level 2 , the Level 1 effect of wake-up time $\left(a_{1 i}\right)$ is a function of the sample average within-person effect of wake-up time $\left(b_{10}\right)$ and a variance component $\left(u_{1 i}\right)$, which allows for individual differences in the magnitude of this effect. All models were estimated with SAS PROC MIXED, with unstructured variance-covariance matrices.

\section{Results}

\section{Descriptive Statistics for Morning Cortisol Levels}

Table 1 shows the descriptive statistics for the raw and lntransformed morning cortisol levels as well as the times these samples were taken. Cortisol levels for the first sample, taken upon waking, were $16.28 \mathrm{nmol} / \mathrm{l}$ and were taken at approximately 6:40 a.m. Cortisol levels for the second sample were $22.79 \mathrm{nmol} / \mathrm{l}$ and were taken at approximately 7:15 a.m. On average, $34 \mathrm{~min}(S D=$ 7.2 ; minimum $=15$, maximum $=60$ ) lapsed between the collection of the two samples. Furthermore, the standard deviations of the cortisol samples and collection times indicated that there was more variability within persons across days in cortisol levels than there was variability between persons in their cortisol levels. Conversely, saliva sample collection times exhibited more variability across individuals (i.e., between persons) than across days (i.e., within persons).

\section{Growth Model of the CAR}

Next, we estimated a three-level multilevel growth model to examine the change in cortisol levels across the morning. Here the intercept for the model reflected the average level of cortisol at the time of the first sampling occasion (i.e., upon waking), and the slope (growth) parameter reflected the amount of change in cortisol per half hour from the time of the first or waking sample. The left column in Table 2 shows that cortisol levels were $16.44 \mathrm{nmol} / \mathrm{l}$ upon waking and increased significantly $(p<.01)$ at a rate of 5.72 $\mathrm{nmol} / \mathrm{l}$ per half hour. In other words, cortisol levels increased by approximately $35 \%$ between Time 1 and Time 2 . The right column

Table 1

Descriptive Statistics for Morning Cortisol Samples and Sample Times

\begin{tabular}{|c|c|c|c|}
\hline \multirow[b]{2}{*}{ Cortisol sample } & \multirow[b]{2}{*}{$M$} & \multicolumn{2}{|c|}{$S D$} \\
\hline & & $\begin{array}{c}\text { Between } \\
\text { persons }\end{array}$ & $\begin{array}{l}\text { Within } \\
\text { persons }\end{array}$ \\
\hline Sample 1 raw & 16.33 & 5.47 & 7.20 \\
\hline Sample 2 raw & 22.76 & 7.33 & 8.73 \\
\hline Sample $1 \ln$ & 2.62 & 0.42 & 0.51 \\
\hline Sample $2 \ln$ & 2.96 & 0.44 & 0.47 \\
\hline Sample 1 time $^{\mathrm{a}}$ & 6.67 & 1.06 & 0.80 \\
\hline Sample 2 time $^{\mathrm{a}}$ & 7.24 & 1.07 & 0.81 \\
\hline Time between samples ${ }^{\mathrm{a}}$ & 0.57 & 0.10 & 0.10 \\
\hline
\end{tabular}

Note. $\quad \ln =$ natural log-transformed value.

${ }^{a}$ Times are shown in military times with decimals reflecting fraction of an hour.
Table 2

Three-Level Multilevel Growth Model Estimate of the Cortisol Awakening Response

\begin{tabular}{|c|c|c|c|c|}
\hline \multirow[b]{2}{*}{ Estimate } & \multicolumn{2}{|c|}{ Raw } & \multicolumn{2}{|c|}{ ln transformed } \\
\hline & Estimate & $S E$ & Estimate & $S E$ \\
\hline \multicolumn{5}{|c|}{ Fixed effects } \\
\hline Intercept & 16.44 & $0.21^{* *}$ & 2.71 & $0.01^{\text {*** }}$ \\
\hline Slope & 5.72 & $0.21^{* *}$ & 0.28 & $0.01^{\text {*** }}$ \\
\hline \multicolumn{5}{|c|}{ Random effects } \\
\hline \multicolumn{5}{|l|}{ Level 3} \\
\hline Intercept & 30.27 & $2.04^{* *}$ & 0.14 & $0.01^{\text {*** }}$ \\
\hline Slope & 96.58 & $8.89^{* *}$ & 0.24 & $0.02^{\text {*** }}$ \\
\hline Covariance & -3.69 & 3.15 & -0.06 & $0.01^{\text {*** }}$ \\
\hline \multicolumn{5}{|l|}{ Level 2} \\
\hline Intercept & 6.93 & $3.49^{*}$ & 0.07 & $0.01^{\text {*** }}$ \\
\hline Slope & 40.23 & $19.84^{*}$ & 0.14 & $0.04^{* * *}$ \\
\hline Covariance & 10.40 & $5.88^{\dagger}$ & -0.05 & $0.01^{\text {*** }}$ \\
\hline \multicolumn{5}{|l|}{ Level 1} \\
\hline Residual & 45.85 & $3.42^{* *}$ & 0.11 & $0.01^{\text {*** }}$ \\
\hline
\end{tabular}

Note. Predicted values are shown.

${ }^{\dagger} p<.10 .{ }^{*} p<.05 .{ }^{* * *} p<.01$.

in Table 2 shows the same model but in natural log-transformed cortisol values and demonstrates that the rate of increase in ln units of cortisol is $.28 \mathrm{nmol} / \mathrm{l}$ per half hour $(p<.01)$. In addition, the results of these models indicated that there was significant variability in both the concentration of cortisol upon waking and the magnitude of the CAR between persons (Level 3 random intercept and slope effects; $p \mathrm{~s}<.01$ ) and within persons across days (Level 2 random intercept and slope effects; $p s<.01$ ). Figure 1 plots the actual mean levels from Table 1 against the predicted values obtained from our three-level multilevel growth curve model. The model provides a good fit to the observed data. Because of the positive skew observed in cortisol values, and consistent with

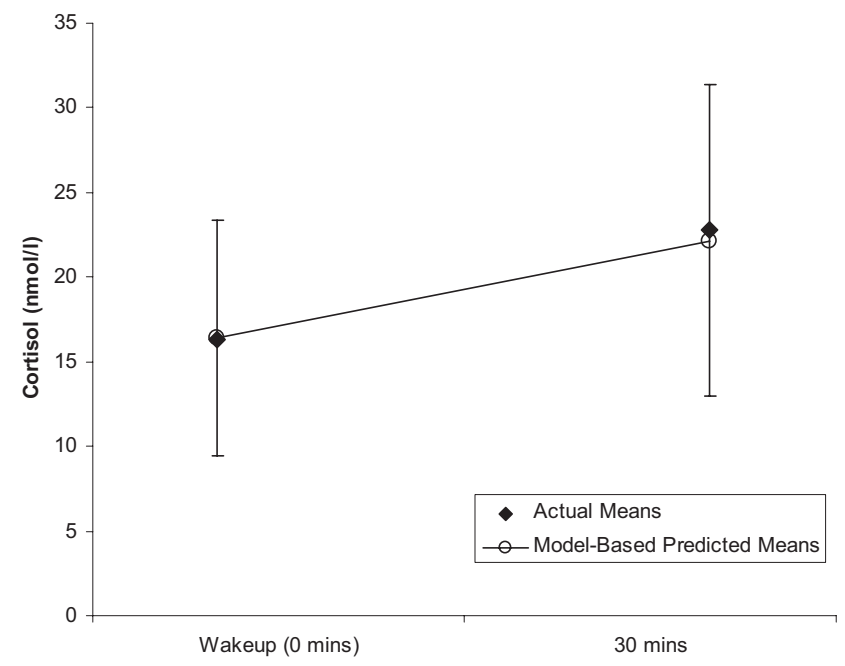

Figure 1. Actual and model-based (predicted values) of morning cortisol levels. Error bars reflect standard deviations. CAR $=$ cortisol awakening response; $\mathrm{y} / \mathrm{o}=$ years old. 
previous research on cortisol (e.g., Adam, Hawkley, Kudielka, \& Cacioppo, 2006), the remaining analyses uses the ln-transformed data.

\section{Stability and Variability in the CAR}

Next, we conducted analyses to examine stability and variability in the CAR, and did so in two ways. First, we examined the correlations in the CAR across days. If the CAR is highly stable, the observed across-day correlations should be high. Second, we decomposed variability in the CAR by estimating an empty multilevel model and calculating the percent of the variability that reflected stable individual differences (i.e., the intraclass correlation coefficient, or ICC). For these analyses, we calculated a CAR value for each person on each day by taking the difference between the two morning cortisol samples and dividing by the duration between the two samples: (lnSample2 - lnSample1)/(TimeSample2 - TimeSample1). Thus, the values reflected in this variable are per-hour changes in lntransformed morning cortisol levels and approximate ordinary least squares estimates of the CAR across individuals and days.

Both sets of analyses evidenced a high degree of intraindividual variability. The correlations in these change scores across days ranged from .13 to .26 (all $p \mathrm{~s}<.01$ ). Next, we used a two-level multilevel model (days nested within persons) to estimate variability across people (i.e., interindividual differences) and across days (i.e., intraindividual variation). To do so, we entered these change scores into an empty two-level multilevel model, the results of which can be seen in Model 1 of Table 3. The sample average CAR was estimated to be $.34(S E=.01)$ ln-units per hour. It is important to note that there was evidence of significant variability across people, as indicated by a significant random intercept (estimate $=.086, S E=.008)$, and across days, as indicated by the significant residual variance component (estimate $=.307, S E=$ .009). The ICC for this model was .219-.086/(.086 + .307) indicating that only about $22 \%$ of the variability in the CAR reflected stable individual differences and the remaining $78 \%$ was accounted for by sources of intraindividual variation. The ICC also indicates that the reliability of measurement of the CAR for discriminating between individuals on any given day is .22 (Snijders \& Bosker, 1999). Reliability, however, increases as a function of the number of assessments per person and can be adjusted accordingly by weighting the ICC by the number of times the variable was assessed. In the current study, the CAR was assessed across 4 days, so the ICC can be recalculated as .086/[.086 + (.307/4)], yielding an estimate of .53 , which is similar to the ICC reported in other studies (e.g., Hellhammer et al., 2007).

\section{Control Variables}

Previous research has shown that cortisol can be influenced by medication use, smoking (Kudielka, Hellhammer, \& Wüst, 2009), and wake-up time (Williams et al., 2005). As such, we estimated a model including these variables as predictors; they were then retained as covariates or control variables in all subsequent models. Because wake-up time was assessed repeatedly, we included both within- and between-persons effects of wake-up time on the CAR, following methods outlined by Hoffman and Stawski (2009). We calculated the within-person effect using person-mean

Table 3

Two-Level Multilevel Models for Age and Gender Differences in Magnitude and Variability in the Cortisol Awakening Response

\begin{tabular}{|c|c|c|c|c|c|c|c|c|c|c|c|c|}
\hline \multirow[b]{2}{*}{ Estimate } & \multicolumn{2}{|c|}{ Model 1} & \multicolumn{2}{|c|}{ Model 2} & \multicolumn{2}{|c|}{ Model 3} & \multicolumn{2}{|c|}{ Model 4} & \multicolumn{2}{|c|}{ Model 5} & \multicolumn{2}{|c|}{ Model 6} \\
\hline & Estimate & $S E$ & Estimate & $S E$ & Estimate & $S E$ & Estimate & $S E$ & Estimate & $S E$ & Estimate & $S E$ \\
\hline \multicolumn{13}{|c|}{ Fixed effects } \\
\hline Intercept & .337 & $.013^{* *}$ & .323 & $.016^{* *}$ & .357 & $.076^{* *}$ & .363 & $.076^{* *}$ & .353 & $.076^{* *}$ & .358 & $.076^{* * *}$ \\
\hline Wake-up time WP & & & -.079 & $.015^{* *}$ & -.079 & $.015^{* *}$ & -.079 & $.015^{* *}$ & -.081 & $.015^{* *}$ & -.081 & $.015^{* *}$ \\
\hline Wake-up time BP & & & -.007 & .011 & -.011 & .011 & -.012 & .011 & -.011 & .011 & -.011 & .011 \\
\hline Medication user $^{\mathrm{a}}$ & & & .015 & .028 & .009 & .029 & .006 & .029 & .007 & .029 & .006 & .028 \\
\hline Smoker $^{\mathrm{b}}$ & & & .086 & $.045^{\dagger}$ & .100 & $.045^{*}$ & .101 & $.045^{*}$ & .101 & $.044^{* *}$ & .101 & $.044^{*}$ \\
\hline Age & & & & & .031 & $.013^{* *}$ & .060 & $.017^{* *}$ & .061 & $.017^{* *}$ & .062 & $.017^{*}$ \\
\hline Gender ${ }^{c}$ & & & & & .075 & $.025^{* *}$ & .074 & $.026^{* *}$ & .074 & $.026^{* *}$ & .075 & $.026^{* *}$ \\
\hline Age $\times$ Gender & & & & & & & -.050 & $.025^{*}$ & -.051 & $.025^{*}$ & -.053 & $.025^{*}$ \\
\hline \multicolumn{13}{|c|}{ Random effects } \\
\hline Intercept & .086 & $.008^{* * *}$ & .093 & $.008^{* *}$ & .090 & $.008^{* *}$ & .089 & $.008^{* *}$ & .084 & $.008^{* *}$ & .083 & $.008^{* * *}$ \\
\hline Wake-up time WP & & & .028 & $.007^{* *}$ & .028 & $.007^{* *}$ & .028 & $.007^{* *}$ & .025 & $.006^{* * *}$ & .024 & $.006^{* *}$ \\
\hline Covariance & & & -.016 & $.006^{* *}$ & -.018 & $.007^{* *}$ & -.018 & $.006^{* *}$ & -.018 & $.006^{* *}$ & -.018 & $.006^{* *}$ \\
\hline Residual & .307 & $.009^{* *}$ & .282 & $.009^{* *}$ & .282 & $.008^{* *}$ & .282 & .009 & .299 & $.048^{* *}$ & .303 & $.048^{* * *}$ \\
\hline Wake-up time BP & & & & & & & & & -.035 & .023 & -.037 & .024 \\
\hline Age & & & & & & & & & .069 & $.027^{* *}$ & .147 & $.043^{* *}$ \\
\hline Gender & & & & & & & & & .324 & $.056^{* *}$ & .328 & $.056^{* *}$ \\
\hline Age $\times$ Gender & & & & & & & & & & & -.132 & $.056^{*}$ \\
\hline \multicolumn{13}{|c|}{ Model fit } \\
\hline-2 log-likelihood & \multicolumn{2}{|c|}{6784.2} & \multicolumn{2}{|c|}{6698.5} & \multicolumn{2}{|c|}{6685.2} & \multicolumn{2}{|c|}{6681.4} & \multicolumn{2}{|c|}{6643.1} & \multicolumn{2}{|c|}{6637.5} \\
\hline
\end{tabular}

Note. Predicted values are shown. $\mathrm{WP}=$ within persons; $\mathrm{BP}=$ between persons.

${ }^{\mathrm{a}} 0=$ did not use medications, $1=$ used medications. $\quad{ }^{\mathrm{b}} 0=$ nonsmoker, $1=$ smoker. $\quad{ }^{\mathrm{c}} 0=$ male, $1=$ female.

${ }^{\dagger} p<.10 .{ }^{*} p<.05$. ${ }^{* *} p<.01$. 
centering, which reflects strictly within-person variation in wake-up time, or variation between when a person woke up and when a person usually wakes up. The within-person effect of wake-up time was also included as a random effect to allow for individual differences in the association between wake-up time and the CAR. The between-persons effect was centered at the sample average wake-up time. The results of this model can be seen in Table 3 (Model 2). The within-person effect of wake-up time was significant, indicating that the CAR was .079 $(S E=.015)$ units shallower for each hour that a person woke up later than usual. The between-persons effect was not significant, which indicates that the magnitude of the CAR did not vary significantly as a function of individual differences in wake-up time. Similarly, medication use did not influence the CAR. Smokers however, did exhibit a slightly steeper CAR, .086 $(S E=.045)$, than nonsmokers.

The bottom portion of Table 3 includes the random effects or variance components. The significant random intercept, .086 $(S E=.008)$, indicates that there were significant individual differences in mean levels of the CAR, whereas the significant random effect of wake-up time, .028 $(S E=.007)$, indicates that there were significant individual differences in the within-person association between wake-up time and the CAR. We also found the residual variance to be significant, $.282(S E=.009)$, indicating that the CAR varied significantly from day to day.

\section{Age and Gender Differences in Level and Variability in the $C A R$}

Next, we expanded the model used to decompose variability in the CAR to consider age and gender differences in both the magnitude of the CAR across the 4 days and the day-to-day variability of the CAR. Such models are highly desirable for examining individual differences with mean and variability as outcomes because both outcomes are predicted simultaneously (Hoffman, 2007). Hence, potential confounding of mean and variability estimates are avoided. ${ }^{1}$

In this set of analyses, we added the main effects of age and gender as predictors of the magnitude of the CAR. As can be seen in Table 3 (Model 3), age and gender were both independent and significant predictors of the CAR after covarying for wake-up time, smoking, and medication use. Women, on average, had a CAR that was $.075(S E=.025, p<.01)$ steeper than men. This indicates that women's CAR was $20 \%$ greater than men's. Similarly, age was positively associated with the CAR such that the slope was $.031(S E=.013, p<.01)$ units steeper per standard deviation $(\sim 12$ years) increase in age. This indicates that a one standard deviation increase in age was associated with a $7 \%$ steeper CAR. In the next model, we added the Age $\times$ Gender interaction to determine whether age effects in the magnitude of the CAR differed between men and women. Model 4 in Table 3 shows that the Age $\times$ Gender interaction was significant (estimate $=-.050, S E=.025, p<.05)$. The interaction indicated that the positive age gradient was significant for men (estimate $=.060$, $S E=.017, p<.01$ ) but not for women (estimate $=.010, S E=$ $.019, p=.60$ ). This interaction is shown in Figure 2A. Together, these models show that the magnitude of the CAR depends on both age and gender. ${ }^{2}$
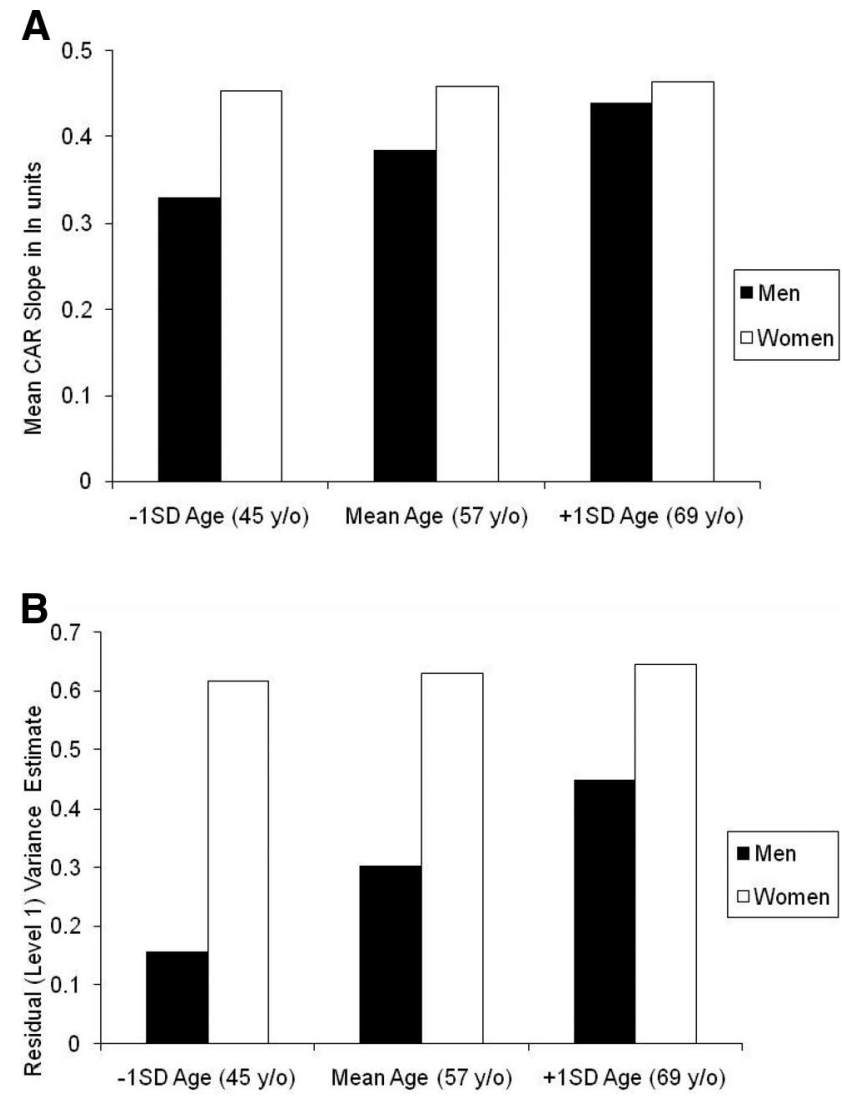

Figure 2. (A) Mean cortisol awakening response (CAR) as a function of age and gender and (B) intraindividual variability in CAR as a function of age and gender.

In the next analyses, we examined age and gender as predictors of the residual variance (day-to-day/within-person variability) in the CAR using methods recommended by Hoffman (2007). The between-persons effect of wake-up time was retained as a covariate when considering age and gender differences in CAR variability. Model 5 in Table 3 shows the results of the model where the between-persons effect of wake-up time, age, and gender were entered simultaneously as predictors of the residual variance. Although the effect of wake-up time was not significant, the effects of both age and gender were significant. The residual variance estimate was $.069(S E=.027, p<.05)$ units greater per standard deviation $(\sim 12$ years $)$ difference in age, and the residual variance estimate for women was $.324(S E=.056, p<.01)$ units higher than the estimate for men. We estimated one final model (Table 3 , Model 6) whereby the Age $\times$ Gender interaction was added as a predictor of the within-person variability. The results of this model

\footnotetext{
${ }^{1}$ We calculated intraindividual means and standard deviations for the CAR variables and examined the correlation, which was $.24(p<.01)$ Thus, our results do not suggest that our findings are due to mean and variability reflecting completely redundant information.

${ }^{2}$ We examined the age and gender effects in the CAR using the three-level growth model, and the overall pattern of results remained unchanged.
} 
show that the Age $\times$ Gender interaction was significant (estimate $=-.132, S E=.056, p<.05)$. The interaction indicated that the positive age gradient for the residual variance was significant for men (estimate $=.147, S E=.043, p<.01$ ) but not for women (estimate $=.014, S E=.037, p=.36$ ), as shown in Figure 2B. Thus, similar to the findings for the magnitude of the CAR, intraindividual variability in the CAR also depended on both age and gender.

\section{Discussion}

Drawing from a national sample of adults between the ages of 33 and 84, the current investigation documented both interindividual differences and intraindividual variability in the CAR. Our first goal was to examine stability and lability of the CAR by comparing interindividual differences and intraindividual variability in this response. Results revealed significant variation in the CAR across persons as well as within persons across days. Our second goal was to examine the extent to which age and gender are associated with both magnitude and intraindividual variability in the CAR across 4 consecutive days. Results revealed a significant Age $\times$ Gender interaction (see Figures $2 \mathrm{~A}$ and $2 \mathrm{~B}$ ) for both magnitude and variability.

Consistent with findings from other community and clinical samples (see reviews by Clow et al., 2004; Fries et al., 2009), our study provides further evidence for a cortisol awakening response. From the time of awakening until $30 \mathrm{~min}$ later, concentrations of salivary cortisol increased by an average of $35 \%$, with values for each time point within the range of those reported across other studies (see review by Clow et al., 2004). The results of the current study also revealed that despite the design parameters of the current study (i.e., 2 samples per day $\times 4$ days), the betweenpersons, or trait-level, reliability of the CAR (.53) was comparable to research with a greater number of samples per day and more days of observation (e.g., Hellhammer et al., 2007). Thus, in the current study, we replicated previous research using a national sample of participants and employing a multiple daily field sampling of salivary cortisol.

\section{Statistical Considerations for Examining the CAR}

Our results indicate a number of important points about using the multilevel model for examining the CAR. First, the multilevel model provided a way to quantify both stability and lability in the CAR and indicated that there is a considerable amount of variability both between and within persons. Second, the growth model applied to examine the magnitude of the CAR provided a very good fit to the observed levels of cortisol as they change throughout the morning. Third, the modeling framework allowed for the simultaneous consideration of age and gender differences in the magnitude of the CAR and intraindividual variability in the CAR, such that mean and variability were not confounded. Finally, this analytic method allows for the inclusion of both time-invariant and time-varying covariates, providing an efficient way to adjust for theoretically important covariates or to rule out third-variable explanations (Hoffman \& Stawski, 2009).

Taken together, the multilevel model is a flexible framework for considering the dynamics of cortisol (i.e., CAR) as well as individual differences in magnitude and variability in the CAR. Such models can be extended to accommodate both time-varying and time-invariant predictors at various levels of analysis, and for both fixed and random effects (i.e., variance components). Although modeling individual differences in fixed effects is commonplace, estimation of heterogeneous variance models, such as those reported in this study, are less common but can be a useful analytic endeavor when prediction of variance is of theoretical importance (cf. Hedeker \& Mermelstein, 2007).

\section{Stability and Intraindividual Variability}

According to our data, there is a high degree of intraindividual variability in the CAR. Estimates of the ICC in our multilevel models indicated that $78 \%$ of total variation in the CAR is due to sources of intraindividual variation. One factor that may partially account for this finding is the impact of wake-up time on the CAR. We did not observe individual differences in wake-up time to be significantly associated with the magnitude of the morning rise, although the direction of the effect was consistent with what has been reported by previous researchers (i.e., later risers tended to have shallower responses; Federenko et al., 2004; see reviews by Fries et al., 2009; Kudielka \& Kirschbaum, 2003; Williams et al., 2005). We did, however, find a significant within-person association between wake-up time and the CAR, indicating that the magnitude of the morning rise was shallower on days when an individual woke up later than his or her average wake-up time. Stalder et al. (2009) reported a similar pattern of findings in their case study of a 57-year-old man who showed higher cortisol levels upon awakening on days when he woke later than usual. The vast differences in the design of the two studies lends further credence to the significance of time of awakening on the CAR.

\section{Age and Gender Findings}

Our findings indicate that variability in men's CAR increases with age, such that older men show greater variability in this response than their younger counterparts. In contrast, women of all ages show consistently high levels of variability in the CAR. We cannot discount the fact that these differences in variability might reflect lack of compliance for those participants who show a more variable response. For example, participants who did not collect their saliva at the allotted times might simply appear to have a more variable response, when, in actuality, no true differences exist. Although this is possible, it begs the question as to why women would be systematically less compliant than men with our research protocol. Given that women tend to be more conscientious than men (Schmitt, Realo, Voracek, \& Allik, 2008), it is doubtful that this explanation fully accounts for the effects shown in the current study. We therefore suggest explanations that take into account gender and age, which may serve as proxies for social role enactment and biological health.

One plausible explanation to our findings lies in how an individual's social roles (e.g., caregiver, laborer) influence his or her expectations of the upcoming day and how these expectations may, in turn, influence his or her HPA functioning. According to Fries et al. (2009), the primary function of the CAR is to prepare an organism for the demands of the upcoming day. Days when challenges are expected should thus be associated with a steeper CAR, a finding that was demonstrated by Rohleder et al. (2007), 
who found that ballroom dancers exhibited a steeper CAR on competition days compared with noncompetition days.

If preparation for the upcoming day is associated with a greater CAR response, perhaps the lability of the CAR is due to social roles and how men and women view the challenges of their upcoming day. Studies indicate that women hold a greater number of qualitatively different social roles, encounter more daily stressors (Almeida \& Kessler, 1998), and are more likely to ruminate over the negative emotions they experience than men (NolenHoeksema \& Corte, 2004). Perhaps, then, women mount a greater response than men on days when they perceive challenges ahead. A study examining gender differences in the CAR on weekdays versus weekends supports this notion. Although men and women's CAR did not differ on weekends, women showed a greater response to awakening on weekdays (Kunz-Ebrecht et al., 2004). This continual fluctuation between days - due to perceived stressors in the environment-may thus translate into greater variability in the CAR for women. Although more research is needed to test this possibility, this hypothesis offers one explanation for the results of the current study.

The aforementioned argument does not explain the age difference found for men, whereby younger men show less variability in their CAR than older men. Perhaps different factors are responsible for younger and older adults' variability in their CAR responses. Among younger adults, women may have a more variable CAR response than men because of social role differences and varying expectations of upcoming days. Among older adults, however, men and women may exhibit increased variability in the CAR for a completely different reason-namely, normative agerelated biological declines. This idea is perhaps best understood within the framework of allostasis and allostatic load. Allostasis is defined as the process of "maintaining stability through change" (McEwen, 2003, p. 10) and is our body's way of coping with the stressors we encounter (McEwen, 2003; Sterling \& Eyer, 1988). After a stressor is perceived, our bodies mount a regulatory response to cope with the ensuing threat. Once the stressor has passed, our systems return to baseline. This continual process of responding to a threat and returning to baseline is useful and effective in the short term but eventually results in wear and tear on the system. This wear and tear is referred to as allostatic load. Because our bodies become more and more taxed due to a lifetime of coping with stressors, allostatic load tends to increase with age (Seeman, Singer, Ryff, Dienberg Love, \& Levy-Storms, 2002). Perhaps, then, day-to-day variability in the CAR response does not reflect proximal environmental factors for older people but instead reflects this wear and tear in the form of an inability to mount a regulatory response because of a compromised HPA axis. Of course, more research is needed to determine whether this idea is a plausible explanation for the findings in the current investigation.

\section{Limitations and Conclusions}

Although this study is informative, it is not without its limitations, most notably not having an objective measure of the time that saliva samples were collected. To accurately assess the CAR, researchers agree that the initial postawakening saliva sample is the most crucial. If, for example, participants do not collect their initial sample immediately after waking, their profiles may appear blunted (Kunz-Ebrecht et al., 2004). Unfortunately, the initial sample is also the most immune to objective indicators of measurement, such as "smart-cap" salivettes. Such devices allow for objective recording of the time a salivette is opened, yet it is impossible to know exactly how much time had passed between when the participant woke up and when the participant provided first sample (Dockray, Bhattacharyya, Molloy, \& Steptoe, 2008). Other objective measures, such as actigraphy, have been employed with success in other studies (e.g., Dockray et al., 2008) but have their own inherent limitations. Although an actigraph allows researchers to identify a participant's wake-up time, it cannot be used to determine when a person takes his or her first sample. Thus, future studies should incorporate the use of both actigraphy and smart-cap salivettes, as the combination of the two-as opposed to one in isolation-would give researchers a much stronger objective measure of compliance.

In addition, it is crucial that study participants understand the importance of complying with the research protocol and fully understand all directions. To this end, we have recently started to provide our participants with videotaped instructions for how to collect, store, and return their saliva samples. This DVD, which is included in the saliva collection kit that is sent to all participants, is available from David M. Almeida upon request.

Although these steps will be undertaken for future research, we recognize the limitations of the current study and understand the concern some researchers have expressed regarding participant compliance (e.g., Kudielka et al., 2002). Thus, until our findings our replicated, we temper the interpretation of some of our conclusions. Notwithstanding these limitations, however, our findings indicate that both the magnitude and day-to-day variability in the CAR are systematically associated with important demographic variables. Although this is a first step, much more work needs to be done before we will be able to determine exactly what variability is and why it may be important for both psychological and physiological health outcomes. A greater understanding of variability in the CAR may perhaps provide a new index by which we can gauge health outcomes.

\section{References}

Adam, E. K., Hawkley, L. C., Kudielka, B. M., \& Cacioppo, J. T. (2006). Day-to-day dynamics of experience-cortisol associations in a population-based sample of older adults. Proceedings of the National Academy of Sciences, USA, 103, 17058-17063.

Almeida, D. M., \& Kessler, R. C. (1998). Everyday stressors and gender differences in daily distress. Journal of Personality and Social Psychology, 75, 670-680.

Almeida, D. M., Wethington, E., \& Kessler, R. C. (2002). The Daily Inventory of Stressful Events: An interview-based approach for measuring daily stressors. Assessment, 9, 41-55.

Bhagwagar, Z., Hafizi, S., \& Cowen, P. J. (2003). Increase in concentration of waking salivary cortisol in recovered patients with depression. American Journal of Psychiatry, 160, 1890-1891.

Chahal, H. S., \& Drake, W. M. (2007). The endocrine system and ageing. Journal of Pathology, 211, 173-180.

Clow, A., Thorn, L., Evans, P., \& Hucklebridge, F. (2004). The awakening cortisol response: Methodological issues and significance. Stress, 7 , $29-37$.

Dahlgren, A., Kecklund, G., Theorell, T., \& Åkerstedt, T. (2009). Day-today variation in saliva cortisol-Relation with sleep, stress and self-rated health. Biological Psychology, 82, 149-155.

de Weerth, C., \& Buitelaar, J. K. (2005). Cortisol awakening response in pregnant women. Psychoneuroendocrinology, 30, 902-907.

Dockray, S., Bhattacharyya, M. R., Molloy, G. J., \& Steptoe, A. (2008). 
The cortisol awakening response in relation to objective and subjective measures of waking in the morning. Psychoneuroendocrinology, 33, $77-82$.

Dressendörfer, R. A., Kirschbaum, C., Rohde, W., Stahl, F., \& Strasburger, C. J. (1992). Synthesis of a cortisol-biotin conjugate and evaluation as a tracer in an immunoassay for salivary cortisol measurement. Journal of Steroid Biochemistry and Molecular Biology, 43, 683-692.

Eizenmann, D. R., Nesselroade, J. R., Featherman, D. L., \& Rowe, J. W. (1997). Intraindividual variability in perceived control in an older sample: The MacArthur successful aging studies. Psychology and Aging, 12, 489-502.

Federenko, I., Wüst, S., Hellhammer, D. H., Dechoux, R., Kumsta, R., \& Kirschbaum, C. (2004). Free cortisol awakening responses are influenced by awakening time. Psychoneuroendocrinology, 29, 174-184.

Fries, E., Dettenborn, L., \& Kirschbaum, C. (2009). The cortisol awakening response (CAR): Facts and future directions. International Journal of Psychophysiology, 72, 67-73.

Geiss, A., Varadi, E., Steinbach, K., Bauer, H. W., \& Anton, F. (1997). Psychoneuroimmunological correlates of persisting sciatic pain in patients who underwent discectomy. Neuroscience Letters, 237, 65-68.

Granger, D. A., Hibel, L. C., Fortunato, C. K., \& Kapelewski, C. H. (2009). Medication effects on salivary cortisol: Tactics and strategy to minimize impact in behavioral and developmental science. Psychoneuroendocrinology, 34, 1437-1448.

Hedeker, D., \& Mermelstein, R. J. (2007). Mixed-effects regression models with heterogeneous variance: Analyzing ecological momentary assessment (EMA) data of smoking. In T. D. Little, J. A. Bovaird, \& N. A. Card (Eds.), Modeling contextual effects in longitudinal studies (pp. 183-206). Mahwah, NJ: Erlbaum.

Hellhammer, J., Fries, E., Schweisthal, O. W., Schlotz, W., Stone, A. A., \& Hagemann, D. (2007). Several daily measurements are necessary to reliably assess the cortisol rise after awakening: State- and trait components. Psychoneuroendocrinology, 32, 80-86.

Hoffman, L. (2007). Multilevel models for examining individual differences in within-person variation and covariation over time. Multivariate Behavioral Research, 42, 609-629.

Hoffman, L., \& Stawski, R. S. (2009). Persons as contexts: Evaluating between-person and within-person effects in longitudinal analysis. $R e-$ search in Human Development, 6, 97-2009.

Hucklebridge, F. H., Clow, A., Abeyguneratne, T., Huezo-Diaz, P., \& Evans, P. (1999). The awakening cortisol response and blood glucose levels. Life Sciences, 64, 931-937.

Kudielka, B. M., Broderick, J. E., \& Kirschbaum, C. (2002). Compliance with saliva sampling protocols: Electronic monitoring reveals invalid cortisol daytime profiles in noncompliant subjects. Psychosomatic Medicine, 65, 313-319.

Kudielka, B. M., Hellhammer, D. H., \& Wüst, S. (2009). Why do we respond so differently? Reviewing determinants of human salivary cortisol responses to challenge. Psychoneuroendocrinology, 34, 2-18.

Kudielka, B. M., \& Kirschbaum, C. (2003). Awakening cortisol responses are influenced by health status and awakening time but not by menstrual cycle phase. Psychoneuroendocrinology, 28, 35-47.

Kunz-Ebrecht, S. R., Kirschbaum, C., Marmot, M., \& Steptoe, A. (2004). Differences in cortisol awakening response on work days and weekends in women and men from the Whitehall II cohort. Psychoneuroendocrinology, 29, 516-528.

McEwen, B. S. (2003). Interacting mediators of allostasis and allostatic load: Towards an understanding of resilience in aging. Metabolism: Clinical and Experimental, 52, 10-16.

Nezlek, J. B. (2008). An introduction to multilevel modeling for social and personality psychology. Social and Personality Psychology Compass, 2, $842-860$

Nolen-Hoeksema, S., \& Corte, C. (2004). Gender and self-regulation. In R. F. Baumeister \& K. D. Vohs (Eds.), Handbook of self-regulation:
Research, theory, and applications (pp. 411-421). New York, NY: Guilford Press.

Polk, D., Cohen, S., Doyle, W. J., Skoner, D. P., \& Kirschbaum, C. (2005). Trait and state affect as predictors of salivary cortisol in healthy adults. Psychoneuroendocrinology, 30, 261-272.

Portella, M. J., Harmer, C. J., Flint, J., Cowen, P., \& Goodwin, G. M. (2005). Enhanced early morning salivary cortisol in neuroticism. American Journal of Psychiatry, 162, 807-809.

Pruessner, J. C., Hellhammer, D. H., \& Kirschbaum, C. (1999). Burnout, perceived stress, and cortisol responses to awakening. Psychosomatic Medicine, 61, 197-204.

Pruessner, J. C., Wolf, O. T., Hellhammer, D. H., Buske-Kirschbaum, A. B., von Auer, K., Jobst, S., .. Kirschbaum, C. (1997). Free cortisol levels after awakening: A reliable biological marker for the assessment of adrenocortical activity. Life Sciences, 61, 2539-2549.

Raudenbush, S. W., \& Bryk, A. S. (2002). Hierarchical linear models: Applications and data analysis methods (2nd ed.). Thousand Oaks, CA: Sage.

Rohleder, N., Beulen, S., Chen, E., Wolf, J. M., \& Kirschbaum, C. (2007) Stress on the dance floor: The cortisol response to social-evaluative threat in competitive ballroom dancers. Personality and Social Psychology Bulletin, 33, 69-84.

Schmitt, D. P., Realo, A., Voracek, M., \& Allik, J. (2008). Why can't a man be more like a woman? Sex differences in Big Five personality traits across 55 cultures. Journal of Personality and Social Psychology, 94, 168-182.

Schulz, P., Kirschbaum, C., Pruessner, J., \& Hellhammer, D. (1998). Increased free cortisol secretion after awakening in chronically stressed individuals due to work overload. Stress Medicine, 14, 91-97.

Seeman, T. E., Singer, B. H., Ryff, C. D., Dienberg Love, G., \& LevyStorms, L. (2002). Social relationships, gender, and allostatic load across two age cohorts. Psychosomatic Medicine, 64, 395-406.

Snijders, T. A. B., \& Bosker, R. J. (1999). Multilevel analysis: An introduction to basic and advanced multilevel modeling. London, England: Sage.

Stalder, T., Hucklebridge, F., Evans, P., \& Clow, A. (2009). Use of a single case study design to examine state variation in the cortisol awakening response: Relationship with time of awakening. Psychoneuroendocrinology, 34, 607-614.

Sterling, P., \& Eyer, J. (1988). Allostasis: A new paradigm to explain arousal pathology. In S. Fisher and J. Reason (Eds.), Handbook of life stress, cognition, and health (pp. 629-649). New York, NY: Wiley.

Thorn, L., Hucklebridge, F., Evans, P., \& Clow, A. (2009). The cortisol awakening response, seasonality, stress and arousal: A study of trait and state influences. Psychoneuroendocrinology, 34, 299-306.

Wessa, M., Rohleder, N., Kirschbaum, C., \& Flor, H. (2006). Altered cortisol awakening response in posttraumatic stress disorder. Psychoneuroendocrinology, 31, 209-215.

Williams, E., Magid, K., \& Steptoe, A. (2005). The impact of time of waking and concurrent subjective stress on the cortisol response to awakening. Psychoneuroendocrinology, 30, 139-148.

Wirtz, P. H., von Känel, R., Emini, L., Ruedisueli, K., Groessbauer, S., Maercker, A., \& Ehlert, U. (2007). Evidence for altered hypothalamuspituitary-adrenal axis functioning in systemic hypertension: Blunted cortisol response to awakening and lower negative feedback sensitivity. Psychoneuroendocrinology, 32, 430-436.

Wüst, S., Wolf, J., Hellhammer, D. H., Federenko, I., Schommer, N., \& Kirschbaum, C. (2000). The cortisol awakening response-normal values and confounds. Noise Health, 2, 79-88.

Received July 28, 2009

Revision received September 28, 2009

Accepted October 5, 2009 